\title{
Potential Blood Biomarkers in Age-related Cerebral Small Vessel Disease
}

\author{
Xin Cheng • Qiang Dong
}

Published online: 28 March 2012

(C) Springer Science+Business Media, LLC 2012

\begin{abstract}
Cerebral small vessel disease is a general term used to reflect clinical, radiological, or pathological phenomena attributed to small vessels of the brain, including small arteries and arterioles but also capillaries and small veins. Age-related cerebral small vessel disease is one of its most common subtypes, and is related to age and vascular risk factors. The consequences of age-related cerebral small vessel disease are mainly lesions located in the subcortical structures such as lacunar infarcts, white matter lesions, large hemorrhages, and microbleeds, which are an important cause of stroke, dementia, and age-related disability. The causes or pathogenesis underlying these phenotypes are not completely understood, and biomarkers to predict the risk and progression are highly needed. This review aims to summarize recent knowledge regarding potential blood biomarkers in age-related cerebral small vessel disease according to the different mechanisms: endothelial dysfunction, inflammation, and $\beta$-amyloid hypothesis.
\end{abstract}

Keywords Cerebral small vessel disease · SVD .

Arteriolosclerosis $\cdot$ Lacunar stroke $\cdot$ Lacunar infarcts ·

White matter lesions $\cdot$ WML $\cdot$ White matter hyperintensities · WMH · Leukoaraiosis · Cerebral microbleeds · CMBs · Biomarker · Endothelial dysfunction · Inflammation . $\beta$-amyloid $\cdot A \beta \cdot$ Cerebral amyloid angiopathy $\cdot$ CAA

X. Cheng $(\triangle) \cdot$ Q. Dong

Department of Neurology and Institute of Neurology,

Huashan Hospital, Fudan University,

12 Wulumuqi Zhong Road,

Shanghai 200040, China

e-mail: chengxincn@hotmail.com

Q. Dong

State Key Laboratory of Medical Neurobiology, Fudan University,

Shanghai, China

\section{Introduction}

"Cerebral small vessel disease" is a general term used to describe clinical, radiological, or pathological phenomena with various etiologies that affect the small arteries, arterioles, venules, and capillaries of the brain $[1 \bullet \bullet, 2 \bullet]$. It is proposed that cerebral small vessel disease (SVD) can be classified into six types according to its different etiologies, which include (1) arteriolosclerosis (or age-related and vascular risk factor-related SVDs); (2) sporadic and hereditary cerebral amyloid angiopathy (CAA); (3) inherited or genetic SVDs distinct from CAA; (4) inflammatory and immunologically mediated SVDs; (5) venous collagenosis; and (6) other SVDs $[1 \bullet \bullet$. Type 1 , arteriolosclerosis, is one of the most prevalent forms among the elderly, together with CAA $[1 \bullet, 3]$. On pathology, loss of smooth muscle cells from the tunica media, deposition of fibrohyaline material, narrowing of the lumen, and thickening of the vessel can be seen, and microatheroma and microaneurysms have been noted in some cases $[4,5]$.

With the aging population and the availability of improved brain imaging techniques, the high prevalence and clinical importance of cerebral SVD have been increasingly recognized in recent years. Advancing age and hypertension are the most important risk factors. Cerebral SVD can be ischemic and/or hemorrhagic, and the principal phenotypes of clinical interest are distinct lacunar infarcts, diffuse cerebral white matter lesions (WMLs, or leukoaraiosis), deep brain hemorrhages, and cerebral microbleeds (CMBs). Growing evidence shows that it is a leading cause of cognitive impairment and functional loss in the elderly population. Therefore, it would be desirable to find some ideal biomarker(s) to predict the risk and progression of cerebral SVD, and there is no doubt that a biomarker derived from a peripheral fluid would be more convenient; thus, this is an 
area of active investigation. This review aims to summarize recent knowledge regarding blood biomarkers in arteriolosclerosis (ie, age-related cerebral SVD).

Quite a few peripheral biomarkers have been proposed to reflect the pathogenesis and correlate with the progression of cerebral SVD, and we classified them here.

\section{Endothelial Dysfunction}

The normal endothelium plays an important role in regulation of cerebral blood flow and autoregulation and in the blood-brain barrier. Endothelial dysfunction is thought to play a pivotal role in the pathogenesis and progression of cerebral SVD, especially in lacunar stroke patients $[6,7 \bullet]$. Endothelial activation is characterized by the increased or de novo expression of leukocyte adhesion molecules (E-selectin, P-selectin, intracellular adhesion molecule-1 [ICAM-1], and vascular cell adhesion molecule-1 [VCAM-1]) and a change of in phenotype from anticoagulant to procoagulant (eg, loss of surface thrombomodulin [TM]) [8, 9•], which can be assessed in vivo by measuring soluble plasma markers that are released into circulation [10]. The different molecules reflect different aspects of endothelial dysfunction.

E-selectin, P-selectin, ICAM-1, TM, Von Willebrand Factor, Tissue Factor

Selectins are transmembrane glycoproteins expressed on activated vascular endothelium (E- and P-selectin) and activated platelets (P-selectin). These adhesion molecules attract leukocytes from the circulation and promote leukocyte rolling along the endothelium [11]. ICAM-1, expressed by activated vascular endothelium and leukocytes, is a precondition for the adhesion and transendothelial migration of leukocytes [12], and increased blood levels reflect an endothelial inflammatory response. TM is a single-chain transmembrane glycoprotein produced and expressed on the endothelial cell surface, where upon binding to thrombin, it not only makes thrombin incapable of generating fibrin clot from fibrinogen, but also activates circulating protein $\mathrm{C}$. Activated protein $\mathrm{C}$, in turn, has an important anticoagulant function including inactivation of clotting factors Va and VIIa [13]. Increased plasma levels of TM are thought to reflect endothelial damage. Von Willebrand factor (vWF) is a large multimeric glycoprotein synthesized by endothelial cells and megakaryocytes that circulates in a noncovalent complex with factor VIII. An increase in plasma vWF is considered to be mainly a marker of endothelial damage as well [14]. Tissue factor (TF) is located at extravascular sites not in contact with the blood but, under stimulation by a variety of agents, TF can be expressed on monocytes and endothelium. By binding to activated factor VIIa, TF initiates the extrinsic coagulation pathway, and levels of TF thus reflect prothrombotic change [15]. The physiological inhibitor of TF is tissue factor pathway inhibitor (TFPI), which is mainly present in endothelial cells and, by binding to activated factor Xa within the TF-VIIa-Xa complex, limits thrombin formation [15].

Several studies [14-20] examined different combinations of these markers in different phases of symptomatic or asymptomatic lacunar stroke (Table 1), and found elevated levels of these circulating markers suggestive of endothelial activation and dysfunction. However, due to the heterogeneity of the studies, robust conclusions are difficult to draw. Moreover, because these studies are cross-sectional, it cannot be established whether endothelial activation is causative or consecutive. Long-term follow-up of patients with intermittent brain magnetic resonance imaging (MRI) might solve this problem. Two prospective studies provide some evidence to support a causal role. After controlling for conventional risk factors and baseline lesion load, plasma ICAM-1 levels increased with the progression of white matter hyperintensities (WMHs) on MRI in a 6-year community-based population of asymptomatic cerebral SVD [21]. In another 6-year longitudinal study [22], serum ICAM-1 levels also were found to be associated with the progression of silent brain infarctions and periventricular WMLs, and this marker may predict impairment in psychomotor function in patients with type 2 diabetes. However, a recent neuropathologic study speaks against cerebral endothelial activation in the pathogenesis of cerebral SVD [23•]. In this case-control study, the authors examined whether brain endothelial ICAM-1 and TM are altered in small penetrating cerebral arteries of pathologically diagnosed cerebral SVD cases, aged control patients without cerebral SVD, young control cases with no brain pathology, and cases with cerebral autosomal dominant arteriopathy with subcortical infarcts and leukoencephalopathy (CADASIL, an early onset hereditary SVD). Interestingly, endothelial ICAM-1 was rarely seen, while robust endothelial TM expression was present in all aged cerebral SVD cases, all aged control brains, and all CADASIL cases [23•]. The findings suggest that elevated blood ICAM-1 [15, 20-22] in cerebral SVD patients was not brain-derived, and may instead derive from peripheral vasculature, while TM being upregulated in response to cerebral SVD, perhaps as a protective mechanism to guard against thrombosis of damaged vessels [23•].

Tissue Plasminogen Activator and Plasminogen Activator Inhibitor-1

Tissue plasminogen activator (tPA), also synthesized and released by endothelial cells, is the primary mediator of 


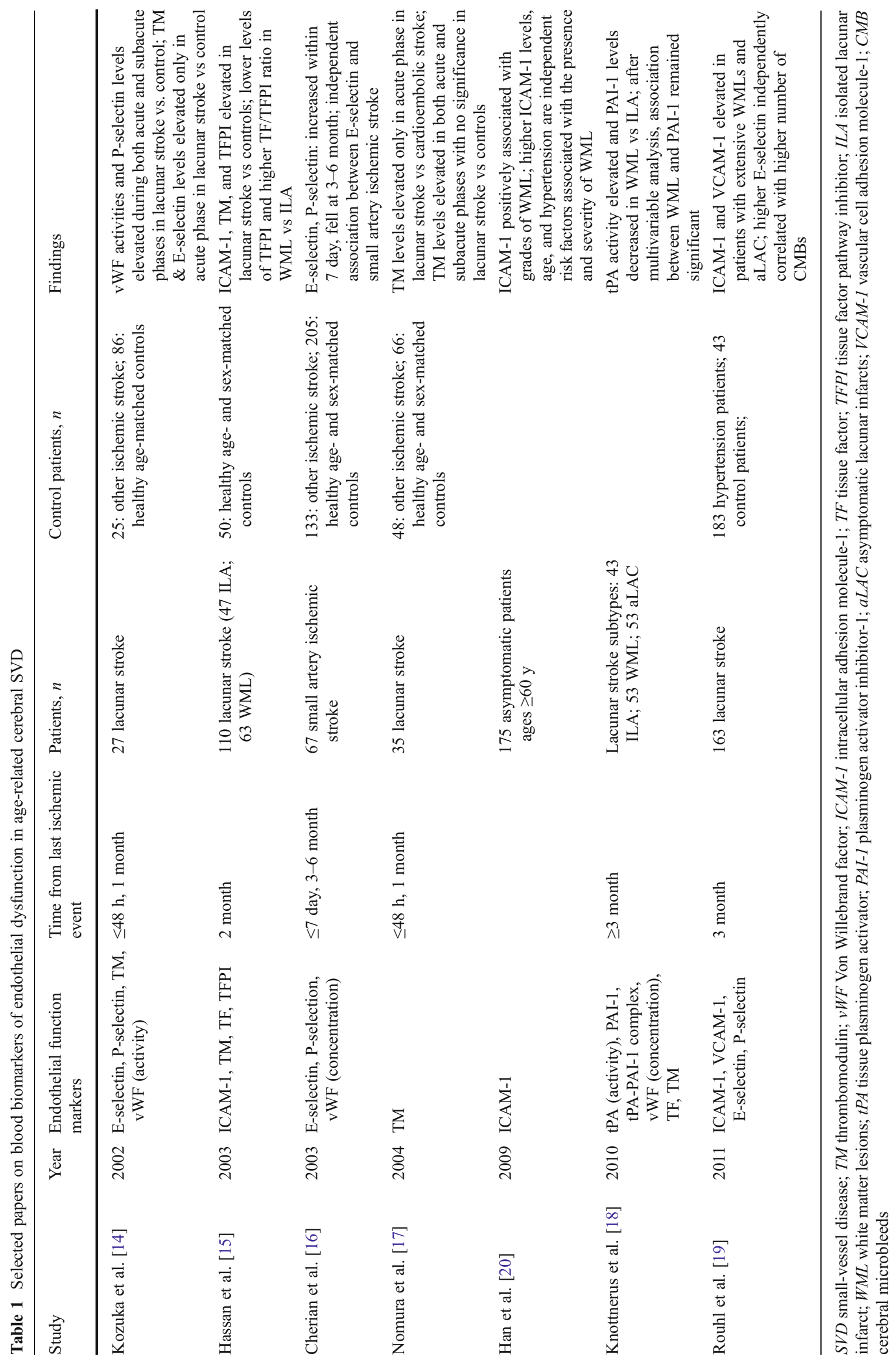


local intravascular fibrinolysis. Vacular injury may induce an acute release of local tPA to mediate the dissolution of arterial thrombus, and a long-term change in the rate of synthesis of tPA, which is a marker of atherosclerotic burden [24]. Therefore, a low capacity for rapid tPA release is likely to predispose to ischemic stroke. The possession of a thymidine $(\mathrm{T})$ allele $(-7351 \mathrm{C} / \mathrm{T}$ polymorphism) is associated with reduced DNA transcription, leading to about half the tPA release observed in those homozygous for the cytosine (C) allele [25]. Because small vessels predominantly express tPA within the brain [26], it is found that the tPA $7351 \mathrm{C} / \mathrm{T}$ polymorphism is an independent risk factor for lacunar stroke [27]. However, levels of tPA were not examined in this study; therefore, the influence of the polymorphism on levels of tPA could not be determined. A recent study showed that higher levels of tPA were found in patients with concomitant extensive WMLs than in those with isolated lacunar infarct, and low levels of plasminogen activator inhibitor-1 (PAI-1), the principal inhibitor of tPA, also were associated with extensive WMLs [18]. Unfortunately, because the study only selected patients with lacunar stroke, levels of tPA among different subtypes of ischemic stroke could not be compared. Moreover, it is a cross-sectional study, and the causal relationship has to be established by long-term follow-up studies.

\section{Homocysteine}

Homocysteine is a dietary sulphur-containing amino acid derived as an intermediate during the metabolism of methionine [28], and genetic factors (most often methylenetetrahydrofolate reductase [MTHFR] C677T polymorphism), systemic disorders (such as renal failure), nutritional status (folate, vitamin B12, and vitamin B6 deficits) or medications can cause hyperhomocysteinemia [29], which can lead to endothelial dysfunction [30]. Therefore, homocysteine may represent an important endothelial toxin in cerebral SVD [6]. Many studies have shown that hyperhomocysteinemia is an independent risk factor for SVD, particularly WMLs [31-50]. Even in patients with symptomatic atherosclerotic (macrovascular) disease, higher homocysteine levels are associated with higher WML volume, presence of lacunar infarcts, and slightly worse cognitive function [51]. After controlling for endothelial dysfunction markers ICAM-1 and TM, the association between homocysteine and SVD was no longer significant, suggesting hyperhomocysteinemia mediates its effect via endothelial dysfunction [37]. It is of particular interest because homocysteine levels can be regulated by folic acid, vitamin B6, and B12, and therefore could be a potential target to prevent stroke [52]. Although homocysteine-lowering treatments in several large randomized trials have thus far failed to provide evidence of benefit for ischemic stroke prevention, a closer look at the data suggests that there may well be an effect in patients with symptomatic cerebral SVD causing lacunar infarction or intracerebral hemorrhage $[52,53 \bullet \bullet]$. Meanwhile, there are no data showing that lowering homocysteine levels improves white matter disease or slows its progression. Larger longitudinal studies in patients with true hyperhomocysteinemia focusing on progression of both lacunar stroke and WMLs are warranted to improve our understanding of the role of homocysteine in the pathophysiology of cerebral SVD.

\section{Asymmetric Dimethylarginine}

Nitric oxide (NO) is one of the most important vasoactive substances released by the endothelium, using the Larginine as substrate. Various pharmacological and physical stimuli lead to the release of NO. Synthesized by endothelial constitutive NO synthase, NO results in vasodilatation and increased blood flow [9•]. Asymmetric dimethylarginine (ADMA) competes with $\mathrm{L}$-arginine as an endogenous non-isoform-specific inhibitor of endothelial NO synthase (eNOS), and it may play a role in endothelial dysfunction $[54,55]$. Therefore, increased plasma ADMA is hypothesized to be a marker for stroke risk prediction and has been associated with other traditional cardiovascular risk factors including hypertension, diabetes, hyperhomocysteinemia, left ventricular hypertrophy, and hypercholesterolemia [56-59]. Furthermore, blood levels of ADMA were elevated in symptomatic SVD patients and correlate with the degree of leukoaraiosis, compared to community control patients free of symptomatic cerebrovascular disease [45]. No association was found between plasma ADMA and homocysteine levels, suggesting that ADMA might be independently associated with SVD [45]. An independent association of the L-arginine/ADMA ratio with microangiopathy-related cerebral damage (lacunar infarction and WMH) also was observed in 712 patients [60]. The Framingham Offspring Study evaluated plasma ADMA concentrations from 2013 stroke-free individuals for whom simultaneous neuroimaging studies were available [61]. ADMA was independently associated with an increased prevalence of silent brain infarcts, most of which (74\% to $86 \%$ ) are lacunar infarcts [62]. Therefore, plasma ADMA may be a potentially useful new biomarker of subclinical vascular brain injury. Because ADMA appears to be a novel biomarker linked to overall cardiovascular mortality, risk of stroke, and endothelial dysfunction, future prospective studies ideally should include control patients with symptomatic atherosclerotic disease (eg, coronary and carotid disease) and asymptomatic control patients with a similar risk factor profile to better elucidate its value as a biomarker in cerebral SVD. 


\section{Endothelial Progenitor Cells}

Endothelial progenitor cells (EPCs) have emerged as a new marker of endothelial dysfunction until recent years. Derived from immature stem cells, EPCs are maturating cells that circulate in peripheral blood. EPCs possess functional and structural characteristics of both stem cells and mature endothelial cells [63]. The number of EPCs can be quantified by cell culture, which depends on the EPC function, and by flow cytometric analysis, which does not depend on the EPC function. Therefore, results are not exchangeable between the two quantification techniques due to this difference [63]. Besides, the function of EPCs is determined by the tests of colony formation, proliferation, and migration among others. EPCs are involved in repair of endothelial damage [63] and are possibly also involved in improving endothelial cell function [64]. Lower EPC numbers and disturbed EPC function relate to different atherosclerotic risk factors [64-66] and a poor prognosis regarding functional recovery $[67,68]$, which reflect a higher consumption of EPCs for restoring the damaged endothelium. Moreover, EPC cluster numbers differed between stroke subtypes: patients with cardioembolic stroke had higher numbers than patients with large or small vessel stroke [68]. Patients with a first-ever lacunar stroke, especially those with silent ischemic lesions, also have impaired cluster-forming potential [69]. Individuals with hypertension with cerebral SVD (WMLs, asymptomatic lacunar infarcts, or CMBs) had lower EPC numbers than control patients with hypertension without cerebral SVD, independently of blood pressure levels [70]. Regulating factors of EPC number or clusterforming potential in cerebral SVD are largely unknown, and recent studies suggest that different haptoglobin phenotypes [69] and angiogenic T cells [70] are likely causative factors. However, the definition of EPC remains controversial [63], and the mentioned studies of EPC in cerebral SVD are all cross-sectional with a relatively small number of patients.

Although the pathogenesis of cerebral SVD remains poorly understood, considerable evidence suggests that endothelial dysfunction, which results in cerebral hypoperfusion and impaired autoregulation and/or increased bloodbrain barrier permeability [71], may play an important role in cerebral SVD. The reviewed blood biomarkers of endothelial dysfunction are found to be related to the severity and even progression of cerebral SVD. However, current data do not confirm that these markers are specific to cerebral SVD because they are involved in cerebral large artery disease as well, and may simply reflect exposure to vascular risk factors and having a stroke. Besides, endothelial function also can be assessed by the vascular response to hypercapnia $\left(\mathrm{CO}_{2}\right)$ or infusion of acetazolamide or L-arginine, or brachial artery flow-mediated dilatation (FMD), as reviewed by Stevenson et al. [72•]. Genetic predisposition involved in endothelial function has been implicated as well (reviewed in [6]). These are beyond the scope of this review.

\section{Inflammation}

Inflammatory processes are implied in the pathogenesis of atherosclerosis [73-75, 76•], and several lines of evidence suggest that inflammation is involved in cerebral SVD as well. The acute-phase reactant C-reactive protein (CRP) has proven to be a sensitive systemic marker of inflammation and to be involved in the endothelial inflammatory response $[77,78]$. The population-based Rotterdam Scan Study of 1033 nondemented elderly individuals showed that higher CRP levels were associated with presence and progression of WMLs, particularly with marked lesion progression, independent of cardiovascular risk factors and carotid atherosclerosis [79]. This finding was later confirmed not only in whites but in blacks as well in the Cardiovascular Health Study [80]. There was no relation between any CRP polymorphism or haplotypes and measures of cerebral SVD [80, 81], suggesting that CRP is not causally involved in the pathogenesis of cerebral SVD. However, several studies failed to build the association between CRP and WMLs, especially in Asian population [82-84], and no significant relationship between CRP levels and WML progression was demonstrated in patients with type 2 diabetes mellitus [22]. The differences may probably be attributed to the different criteria for classification of WMLs, different ages of study population, and racial differences. On the other hand, in the Rotterdam Scan Study, higher plasma CRP levels were marginally associated with greater prevalence and incidence of lacunar infarcts [79]. In two independent cohorts of elderly Japanese individuals, CRP levels were elevated in patients with silent brain infarctions [85, 86]. Moreover, baseline CRP levels were significantly associated with progression of silent brain infarctions at 3-year follow-up in patients with type 2 diabetes mellitus [22]. Thus, elevated CRP levels may be related to WMLs and lacunar infarcts via different mechanisms. More recently, elevated CRP levels were found to be associated with CMBs, another important manifestation of cerebral SVD, independent of cardiovascular risk factors, carotid atherosclerosis, WMLs, and silent lacunar infarcts [87].

As a main inducer of hepatic production of CRP, interleukin-6 (IL-6) is a multifunctional cytokine that plays important roles in the regulation of the immune responses and inflammation. Same as CRP, IL-6 could predict future risk of recurrent ischemic stroke and cardiovascular events $[88,89]$. Besides, the Cardiovascular Health Study showed that plasma IL-6 levels were significantly associated with the presence of WMLs and brain infarcts in whites and blacks, while common haplotypes of the IL- 6 gene were 
significantly associated with WMLs and brain infarcts only in whites [80]. IL-6 levels also were found to be independently related with CMBs [87].

Other markers of inflammation, such as monocyte [90] and leukocyte [84] counts, monocyte/macrophage activation (neopterin) [19], and myeloperoxidase (MPO) [91], have been identified to independently predict the presence of lacunar infarcts or WMLs. Although markers of inflammation are not disease-specific but are sensitive markers produced in response to tissue injury, infectious agents, immunological stimuli, and inflammation, the characteristics desired of inflammatory markers of cardiovascular diseases are as follows: independence from established risk factors, well-established associations with cardiovascular diseases in observational studies and clinical trials, the availabilities of population norms to guide the interpretation of results, the applicabilities of results to different population groups, the availability of a standardized assay with acceptable reproducibility, and an acceptable assay cost [92]. Hopefully, the ongoing Levels of Inflammatory Markers in the Treatment of Stroke (LIMITS) study [93] will identify novel inflammatory biomarkers with the aforementioned characteristics for use in risk prediction and treatment selection in patients with cerebral SVD.

\section{ß-Amyloid Hypothesis}

Plasma $\beta$-amyloid peptide $(A \beta)$ is a peptide consisting of either $42\left(A \beta_{1-42}\right)$ or $40\left(A \beta_{1-40}\right)$ amino acids derived from a proteolytic processing of the amyloid precursor protein. Insoluble $A \beta$ fibrils are the predominant constituents of senile plaques, one of the pathological hallmarks of $\mathrm{AD}$, and of cerebrovascular amyloid in the related condition of CAA. Plaque amyloid is primarily comprised of $A \beta_{42}$, whereas vascular amyloid is formed by $A \beta_{40}$ species [94]. $A \beta$ has emerged as a potential mediator of microvascular dysfunction. In vitro studies have suggested direct physiologic or toxic effects of $A \beta$ on the blood vessel wall [95]. Plasma $A \beta_{40}$ and $A \beta_{42}$ levels are strongly associated with the presence of lacunar infarcts and severity of WMLs in nondemented elderly patients who carry an apolipoprotein E (APOE) $\varepsilon 4$ allele in the Rotterdam cohort [96]. In patients with $\mathrm{AD}$, mild cognitive impairment (MCI), or CAA, plasma $A \beta_{40}$ concentration is independently associated with extent of WMHs [97]. Furthermore, a recent study demonstrated that plasma $A \beta_{40}$ levels, but not $A \beta_{42}$, are strongly associated with the diffuse SVD subtype (ie, leukoaraiosis [98]). Plasma $A \beta_{40}$ levels are also found to be markedly elevated in patients with ischemic stroke, and patients with cardioembolic and large-artery atherosclerotic infarcts had higher levels than those with SVD infarctions [99]. However, this study included only 12 patients classified as cerebral SVD and the presence of WMLs was not analyzed.

Taken together, both experimental and clinical studies have suggested the pathophysiological role of $A \beta_{40}$ in disrupting microvascular function, and circulating $A \beta$ might be a novel marker of cerebral SVD. Prospective studies are needed to confirm its role. Moreover, because AD and cerebral SVD, especially age-related cerebral SVD, usually coexist in elderly patients, it also would be interesting to compare plasma $A \beta$ levels in patients with vascular cognitive impairment with no dementia (VCI-ND), vascular dementia $(\mathrm{VaD}), \mathrm{MCI}$, and $\mathrm{AD}$ in future studies.

\section{Conclusions}

Age-related cerebral SVD is a common condition in elderly patients and has been strongly associated with risk of incident stroke, dementia, and age-related disability. Endothelial dysfunction, inflammation, and $A \beta$ are suggested to be involved in the pathogenesis of cerebral SVD. Although the investigations of blood biomarkers regarding these mechanisms are quite diverse and active, uniform and robust conclusions are difficult to draw due to the heterogeneity of the studies. Future studies should precisely classify the different phenotypes of cerebral SVD using established clinical criteria combined with MRI findings, include symptomatic control patients with atherosclerotic disease and asymptomatic control patients with similar risk factor profiles, and follow up patients with repeated MRI and blood collection. Meanwhile, more attention and targeted efforts are needed to identify novel biochemical biomarkers that shed light on the multifactorial pathogenesis and offer potential therapeutic targets.

Acknowledgements This work was supported by grants from the National Natural Science Foundation of China 811008861 (XC) and Specialized Research Fund for the Doctoral Program of Higher Education 20100071120081 (XC).

\section{References}

Papers of particular interest, published recently, have been highlighted as follows:

- Of importance

•. Of major importance

1. •• Pantoni L. Cerebral small vessel disease: from pathogenesis and clinical characteristics to therapeutic challenges. Lancet Neurol. 2010;9(7):689-701. This comprehensive review summarized the classification, pathogenesis, and neuroimaging and therapeutic aspects of cerebral SVD, providing some basic concepts with a modern overview to enable understanding of recent progress and future directions in the field. 
2. - Moran C, Phan TG, Srikanth VK. Cerebral small vessel disease: a review of clinical, radiological, and histopathological phenotypes. Int J Stroke. 2012;7(1):36-46. This recent review summarized recent knowledge regarding different phenotypes of cerebral SVD and placed it in context with classical clinicopathological observations to provide mechanistic, clinical, and therapeutic insights into cerebral SVD.

3. Grinberg LT, Thal DR. Vascular pathology in the aged human brain. Acta Neuropathol. 2010;119(3):277-90.

4. Fisher CM. The arterial lesions underlying lacunes. Acta Neuropathol. 1968;12(1):1-15.

5. Fisher CM. Capsular infarcts: the underlying vascular lesions. Arch Neurol. 1979;36(2):65-73.

6. Markus HS. Genes, endothelial function and cerebral small vessel disease in man. Exp Physiol. 2008;93(1):121-7.

7. - Farrall AJ, Wardlaw JM. Blood-brain barrier: ageing and microvascular disease-systematic review and meta-analysis. Neurobiol Aging 2009;30(3):337-52. This paper systematically reviewed the literature and found evidence that blood-brain barrier (BBB) permeability increased in normal aging and further increased in patients with dementia (especially vascular dementia) and with increasing WMLs.

8. Deanfield JE, Halcox JP, Rabelink TJ. Endothelial function and dysfunction: testing and clinical relevance. Circulation. 2007;115 (10):1285-95.

9. - Knottnerus IL, Ten Cate H, Lodder J, et al. Endothelial dysfunction in lacunar stroke: a systematic review. Cerebrovasc Dis 2009;27(5):519-26. This paper systematically reviewed the evidence of endothelial activation and dysfunction in lacunar stroke, and concluded that endothelial dysfunction might be involved in the pathogenesis of lacunar stroke, especially in those patients with concomitant silent lacunar infarcts and WMLs.

10. Cines DB, Pollak ES, Buck CA, et al. Endothelial cells in physiology and in the pathophysiology of vascular disorders. Blood. 1998;91(10):3527-61.

11. Springer TA. Adhesion receptors of the immune system. Nature. 1990;346(6283):425-34.

12. Bevilacqua MP, Stengelin S, Gimbrone Jr MA, Seed B. Endothelial leukocyte adhesion molecule 1: an inducible receptor for neutrophils related to complement regulatory proteins and lectins. Science. 1989;243(4895):1160-5.

13. Fisher M. Thrombomodulin and the brain: past, present, and future. Neurology. 2012;78(3):157-8.

14. Kozuka K, Kohriyama T, Nomura E, et al. Endothelial markers and adhesion molecules in acute ischemic stroke-sequential change and differences in stroke subtype. Atherosclerosis. 2002;161 (1):161-8.

15. Hassan A, Hunt BJ, O'Sullivan M, et al. Markers of endothelial dysfunction in lacunar infarction and ischaemic leukoaraiosis. Brain. 2003;126(Pt 2):424-32.

16. Cherian P, Hankey GJ, Eikelboom JW, et al. Endothelial and platelet activation in acute ischemic stroke and its etiological subtypes. Stroke. 2003;34(9):2132-7.

17. Nomura E, Kohriyama T, Kozuka K, et al. Significance of serum soluble thrombomodulin level in acute cerebral infarction. Eur J Neurol. 2004;11(5):329-34.

18. Knottnerus IL, Govers-Riemslag JW, Hamulyak K, et al. Endothelial activation in lacunar stroke subtypes. Stroke. 2010;41(8):1617-22.

19. Rouhl RP, Damoiseaux JG, Lodder J, et al. Vascular inflammation in cerebral small vessel disease. Neurobiol Aging 2011.

20. Han JH, Wong KS, Wang YY, et al. Plasma level of sICAM-1 is associated with the extent of white matter lesion among asymptomatic elderly subjects. Clin Neurol Neurosurg. 2009;111 (10):847-51.

21. Markus HS, Hunt B, Palmer K, et al. Markers of endothelial and hemostatic activation and progression of cerebral white matter hyperintensities: longitudinal results of the Austrian Stroke Prevention Study. Stroke. 2005;36(7):1410-4.

22. Umemura T, Kawamura T, Umegaki H, et al. Endothelial and inflammatory markers in relation to progression of ischaemic cerebral small-vessel disease and cognitive impairment: a 6-year longitudinal study in patients with type 2 diabetes mellitus. J Neurol Neurosurg Psychiatry. 2011;82(11):1186-94.

23. - Giwa MO, Williams J, Elderfield K, et al. Neuropathologic evidence of endothelial changes in cerebral small vessel disease. Neurology 2012;78(3):167-74. This case-control postmortem study examined the expression of brain endothelial ICAM-1, TM, and IL-6 in small penetrating cerebral arteries of pathologically diagnosed SVD cases and different control patients to test whether endothelial activation and dysfunction is causative or consecutive in the pathogenesis of cerebral SVD. The study confirms endothelial abnormality in SVD, but speaks against its causative role.

24. Kooistra T, Schrauwen Y, Arts J, Emeis JJ. Regulation of endothelial cell t-PA synthesis and release. Int J Hematol. 1994;59 (4):233-55.

25. Ladenvall $P$, Wall $U$, Jern $S$, Jern C. Identification of eight novel single-nucleotide polymorphisms at human tissue-type plasminogen activator (t-PA) locus: association with vascular t-PA release in vivo. Thromb Haemost. 2000;84(2):150-5.

26. Levin EG, del Zoppo GJ. Localization of tissue plasminogen activator in the endothelium of a limited number of vessels. Am J Pathol. 1994;144(5):855-61.

27. Jannes J, Hamilton-Bruce MA, Pilotto L, et al. Tissue plasminogen activator $-7351 \mathrm{C} / \mathrm{T}$ enhancer polymorphism is a risk factor for lacunar stroke. Stroke. 2004;35(5):1090-4.

28. Hankey GJ, Eikelboom JW. Homocysteine and vascular disease. Lancet. 1999;354(9176):407-13.

29. Welch GN, Loscalzo J. Homocysteine and atherothrombosis. N Engl J Med. 1998;338(15):1042-50.

30. Woo KS, Chook P, Lolin YI, et al. Hyperhomocyst(e)inemia is a risk factor for arterial endothelial dysfunction in humans. Circulation. 1997;96(8):2542-4.

31. Fassbender K, Mielke O, Bertsch T, et al. Homocysteine in cerebral macroangiography and microangiopathy. Lancet. 1999;353 (9164):1586-7.

32. Eikelboom JW, Hankey GJ, Anand SS, et al. Association between high homocyst(e)ine and ischemic stroke due to large- and smallartery disease but not other etiologic subtypes of ischemic stroke. Stroke. 2000;31(5):1069-75.

33. Matsui T, Arai H, Yuzuriha T, et al. Elevated plasma homocysteine levels and risk of silent brain infarction in elderly people. Stroke. 2001;32(5):1116-9.

34. Vermeer SE, van Dijk EJ, Koudstaal PJ, et al. Homocysteine, silent brain infarcts, and white matter lesions: The Rotterdam Scan Study. Ann Neurol. 2002;51(3):285-9.

35. Dufouil C, Alperovitch A, Ducros V, Tzourio C. Homocysteine, white matter hyperintensities, and cognition in healthy elderly people. Ann Neurol. 2003;53(2):214-21.

36. Longstreth Jr WT, Katz R, Olson J, et al. Plasma total homocysteine levels and cranial magnetic resonance imaging findings in elderly persons: the Cardiovascular Health Study. Arch Neurol. 2004;61(1):67-72.

37. Hassan A, Hunt BJ, O'Sullivan M, et al. Homocysteine is a risk factor for cerebral small vessel disease, acting via endothelial dysfunction. Brain. 2004;127(Pt 1):212-9.

38. Sachdev P, Parslow R, Salonikas C, et al. Homocysteine and the brain in midadult life: evidence for an increased risk of leukoaraiosis in men. Arch Neurol. 2004;61(9):1369-76.

39. Scott TM, Tucker KL, Bhadelia A, et al. Homocysteine and B vitamins relate to brain volume and white-matter changes in geriatric patients with psychiatric disorders. Am J Geriatr Psychiatry. 2004;12(6):631-8. 
40. Iso H, Moriyama $Y$, Sato $S$, et al. Serum total homocysteine concentrations and risk of stroke and its subtypes in Japanese. Circulation. 2004;109(22):2766-72.

41. Wright CB, Paik MC, Brown TR, et al. Total homocysteine is associated with white matter hyperintensity volume: the Northern Manhattan Study. Stroke. 2005;36(6):1207-11.

42. Perini F, Galloni E, Bolgan I, et al. Elevated plasma homocysteine in acute stroke was not associated with severity and outcome: stronger association with small artery disease. Neurol Sci. 2005;26(5):310-8.

43. Wong A, Mok V, Fan YH, et al. Hyperhomocysteinemia is associated with volumetric white matter change in patients with small vessel disease. J Neurol. 2006;253(4):441-7.

44. Censori B, Partziguian T, Manara O, Poloni M. Plasma homocysteine and severe white matter disease. Neurol Sci. 2007;28(5):259-63.

45. Khan U, Hassan A, Vallance P, Markus HS. Asymmetric dimethylarginine in cerebral small vessel disease. Stroke. 2007;38(2):411-3.

46. Seshadri S, Wolf PA, Beiser AS, et al. Association of plasma total homocysteine levels with subclinical brain injury: cerebral volumes, white matter hyperintensity, and silent brain infarcts at volumetric magnetic resonance imaging in the Framingham Offspring Study. Arch Neurol. 2008;65(5):642-9.

47. Khan U, Crossley C, Kalra L, et al. Homocysteine and its relationship to stroke subtypes in a UK black population: the south London ethnicity and stroke study. Stroke. 2008;39(11):2943-9.

48. Tseng YL, Chang YY, Liu JS, et al. Association of plasma homocysteine concentration with cerebral white matter hyperintensity on magnetic resonance images in stroke patients. J Neurol Sci. 2009;284(1-2):36-9.

49. Oncel C, Demir S, Guler S, et al. Association between cholesterols, homocysteine and silent brain infarcts. Intern Med J. 2009;39 (3):150-5

50. Pavlovic AM, Pekmezovic T, Obrenovic R, et al. Increased total homocysteine level is associated with clinical status and severity of white matter changes in symptomatic patients with subcortical small vessel disease. Clin Neurol Neurosurg. 2011;113(9):711-5.

51. Kloppenborg RP, Nederkoorn PJ, van der Graaf Y, Geerlings MI. Homocysteine and cerebral small vessel disease in patients with symptomatic atherosclerotic disease. The SMART-MR study. Atherosclerosis. 2011;216(2):461-6.

52. Spence JD. Homocysteine-lowering therapy: a role in stroke prevention? Lancet Neurol. 2007;6(9):830-8.

53. $\cdot$ B vitamins in patients with recent transient ischaemic attack or stroke in the VITAmins TO Prevent Stroke (VITATOPS) trial: a randomised, double-blind, parallel, placebo-controlled trial. Lancet Neurol 2010;9(9):855-65. This randomized, double-blind, placebo-controlled and investigator-driven trial examined whether the addition of once-daily supplements of B vitamins would lower total homocysteine and reduce major vascular events and death in patients with recent stroke or transient ischemic attack. Although the overall results were negative, prespecified subgroup analysis showed patients with symptomatic cerebral SVD causing lacunar infarction or intracerebral hemorrhage might benefit from $B$ vitamins.

54. Boger RH, Bode-Boger SM, Szuba A, et al. Asymmetric dimethylarginine (ADMA): a novel risk factor for endothelial dysfunction: its role in hypercholesterolemia. Circulation. 1998;98(18):1842-7.

55. Lentz SR, Rodionov RN, Dayal S. Hyperhomocysteinemia, endothelial dysfunction, and cardiovascular risk: the potential role of ADMA. Atheroscler Suppl. 2003;4(4):61-5.

56. Yoo JH, Lee SC. Elevated levels of plasma homocyst(e)ine and asymmetric dimethylarginine in elderly patients with stroke. Atherosclerosis. 2001;158(2):425-30.

57. Wanby P, Teerlink T, Brudin L, et al. Asymmetric dimethylarginine (ADMA) as a risk marker for stroke and TIA in a Swedish population. Atherosclerosis. 2006;185(2):271-7.
58. Leong T, Zylberstein D, Graham I, et al. Asymmetric dimethylarginine independently predicts fatal and nonfatal myocardial infarction and stroke in women: 24-year follow-up of the population study of women in Gothenburg. Arterioscler Thromb Vasc Biol. 2008;28(5):961-7.

59. Saenger AK, Christenson RH. Stroke biomarkers: progress and challenges for diagnosis, prognosis, differentiation, and treatment Clin Chem. 2010;56(1):21-33.

60. Notsu Y, Nabika T, Bokura H, et al. Evaluation of asymmetric dimethylarginine and homocysteine in microangiopathy-related cerebral damage. Am J Hypertens. 2009;22(3):257-62.

61. Pikula A, Boger RH, Beiser AS, et al. Association of plasma ADMA levels with MRI markers of vascular brain injury: Framingham offspring study. Stroke. 2009;40(9):2959-64.

62. Masuda J, Nabika T, Notsu Y. Silent stroke: pathogenesis, genetic factors and clinical implications as a risk factor. Curr Opin Neurol. 2001;14(1):77-82.

63. Rouhl RP, van Oostenbrugge RJ, Damoiseaux J, et al. Endothelial progenitor cell research in stroke: a potential shift in pathophysiological and therapeutical concepts. Stroke. 2008;39(7):2158-65.

64. Hill JM, Zalos G, Halcox JP, et al. Circulating endothelial progenitor cells, vascular function, and cardiovascular risk. N Engl J Med. 2003;348(7):593-600.

65. Ghani U, Shuaib A, Salam A, et al. Endothelial progenitor cells during cerebrovascular disease. Stroke. 2005;36(1):151-3.

66. Werner N, Kosiol S, Schiegl T, et al. Circulating endothelial progenitor cells and cardiovascular outcomes. N Engl J Med. 2005;353(10):999-1007.

67. Sobrino T, Hurtado O, Moro MA, et al. The increase of circulating endothelial progenitor cells after acute ischemic stroke is associated with good outcome. Stroke. 2007;38(10):2759-64.

68. Chu K, Jung KH, Lee ST, et al. Circulating endothelial progenitor cells as a new marker of endothelial dysfunction or repair in acute stroke. Stroke. 2008;39(5):1441-7.

69. Rouhl RP, van Oostenbrugge RJ, Damoiseaux JG, et al. Haptoglobin phenotype may alter endothelial progenitor cell cluster formation in cerebral small vessel disease. Curr Neurovasc Res. 2009;6 (1):32-41.

70. Rouhl RP, Mertens AE, van Oostenbrugge RJ, et al. Angiogenic Tcells and putative endothelial progenitor cells in hypertensionrelated cerebral small vessel disease. Stroke. 2012;43(1):256-8.

71. Wardlaw JM, Sandercock PA, Dennis MS, Starr J. Is breakdown of the blood-brain barrier responsible for lacunar stroke, leukoaraiosis, and dementia? Stroke. 2003;34(3):806-12.

72. • Stevenson SF, Doubal FN, Shuler K, Wardlaw JM. A systematic review of dynamic cerebral and peripheral endothelial function in lacunar stroke versus controls. Stroke 2010;41(6):e434-442. The paper systematically assessed all relevant studies of dynamic endothelial function in patients with lacunar stroke and controlled for risk factor exposure and other stroke subtypes. The authors concluded that current data do not confirm that endothelial dysfunction is specific to small vessel stroke, and suggested that future stroke studies should include control subjects with nonlacunar stroke.

73. Ross R. Atherosclerosis-an inflammatory disease. N Engl J Med. 1999;340(2):115-26.

74. Libby P. Inflammation in atherosclerosis. Nature. 2002;420 (6917):868-74

75. Rost NS, Wolf PA, Kase CS, et al. Plasma concentration of Creactive protein and risk of ischemic stroke and transient ischemic attack: the Framingham study. Stroke. 2001;32(11):2575-9.

76. - Kaptoge S, Di Angelantonio E, Lowe G, et al. C-reactive protein concentration and risk of coronary heart disease, stroke, and mortality: an individual participant meta-analysis. Lancet 2010;375 (9709):132-40. This meta-analysis assessed the associations of CRP concentration with risk of vascular and nonvascular 
outcomes. The results showed that CRP concentration has continuous associations with the risk of ischemic stroke, and such associations depend on conventional risk factors and fibrinogen concentration.

77. Ridker PM, Hennekens CH, Buring JE, Rifai N. C-reactive protein and other markers of inflammation in the prediction of cardiovascular disease in women. N Engl J Med. 2000;342(12):836-43.

78. Khreiss T, Jozsef L, Potempa LA, Filep JG. Conformational rearrangement in C-reactive protein is required for proinflammatory actions on human endothelial cells. Circulation. 2004;109(16):201622.

79. van Dijk EJ, Prins ND, Vermeer SE, et al. C-reactive protein and cerebral small-vessel disease: the Rotterdam Scan Study. Circulation. 2005;112(6):900-5.

80. Fornage M, Chiang YA, O'Meara ES, et al. Biomarkers of inflammation and MRI-Defined Small Vessel Disease of the brain: the Cardiovascular Health Study. Stroke. 2008;39(7):1952-9.

81. Reitz C, Berger K, de Maat MP, et al. CRP gene haplotypes, serum CRP, and cerebral small-vessel disease: the Rotterdam Scan Study and the MEMO Study. Stroke. 2007;38(8):2356-9.

82. Schmidt R, Schmidt H, Pichler M, et al. C-reactive protein, carotid atherosclerosis, and cerebral small-vessel disease: results of the Austrian Stroke Prevention Study. Stroke. 2006;37(12):2910-6.

83. Wada M, Nagasawa $H$, Kurita K, et al. Cerebral small vessel disease and C-reactive protein: results of a cross-sectional study in community-based Japanese elderly. J Neurol Sci. 2008;264(12):43-9.

84. Kim CK, Lee SH, Kim BJ, et al. Elevated leukocyte count in asymptomatic subjects is associated with a higher risk for cerebral white matter lesions. Clin Neurol Neurosurg. 2011;113(3):177-80.

85. Hoshi T, Kitagawa K, Yamagami H, et al. Relations of serum highsensitivity C-reactive protein and interleukin-6 levels with silent brain infarction. Stroke. 2005;36(4):768-72.

86. Ishikawa J, Tamura Y, Hoshide S, et al. Low-grade inflammation is a risk factor for clinical stroke events in addition to silent cerebral infarcts in Japanese older hypertensives: the Jichi Medical School ABPM Study, wave 1. Stroke. 2007;38(3):911-7.

87. Miwa K, Tanaka M, Okazaki S, et al. Relations of blood inflammatory marker levels with cerebral microbleeds. Stroke. 2011;42 (11):3202-6.
88. Welsh P, Lowe GD, Chalmers J, et al. Associations of proinflammatory cytokines with the risk of recurrent stroke. Stroke. 2008;39 (8):2226-30.

89. Patterson CC, Smith AE, Yarnell JW, et al. The associations of interleukin-6 (IL-6) and downstream inflammatory markers with risk of cardiovascular disease: the Caerphilly Study. Atherosclerosis. 2010;209(2):551-7.

90. Cortina MG, Campello AR, Conde JJ, et al. Monocyte count is an underlying marker of lacunar subtype of hypertensive small vessel disease. Eur J Neurol. 2008;15(7):671-6.

91. Wright CB, Moon Y, Paik MC, et al. Inflammatory biomarkers of vascular risk as correlates of leukoariosis. Stroke. 2009;40 (11):3466-71.

92. Pearson TA, Mensah GA, Alexander RW, et al. Markers of inflammation and cardiovascular disease: application to clinical and public health practice: a statement for healthcare professionals from the Centers for Disease Control and Prevention and the American Heart Association. Circulation. 2003;107(3):499 511.

93. Elkind MS, Luna JM, Coffey CS, et al. The Levels of Inflammatory Markers in the Treatment of Stroke study (LIMITS): inflammatory biomarkers as risk predictors after lacunar stroke. Int J Stroke. 2010;5(2):117-25.

94. Selkoe DJ. Alzheimer's disease: genes, proteins, and therapy. Physiol Rev. 2001;81(2):741-66.

95. Thomas T, Thomas G, McLendon C, et al. beta-Amyloid-mediated vasoactivity and vascular endothelial damage. Nature. 1996;380 (6570):168-71.

96. van Dijk EJ, Prins ND, Vermeer SE, et al. Plasma amyloid beta, apolipoprotein E, lacunar infarcts, and white matter lesions. Ann Neurol. 2004;55(4):570-5.

97. Gurol ME, Irizarry MC, Smith EE, et al. Plasma beta-amyloid and white matter lesions in $\mathrm{AD}, \mathrm{MCI}$, and cerebral amyloid angiopathy. Neurology. 2006;66(1):23-9.

98. Gomis M, Sobrino T, Ois A, et al. Plasma beta-amyloid 1-40 is associated with the diffuse small vessel disease subtype. Stroke. 2009;40(10):3197-201.

99. Lee PH, Bang OY, Hwang EM, et al. Circulating beta amyloid protein is elevated in patients with acute ischemic stroke. J Neural Transm. 2005;112(10):1371-9. 